\title{
Paciente en programa de diálisis y arteriopatía periférica
}

José María Lancho Casares - Trinidad Juan Larma - Concha de Vega Jiménez - Manolo Nieto Guerrero Josefina Acedo Agúndez

Hospital San Pedro de Alcántara. Cáceres

\section{Resumen}

La alta prevalencia de enfermedad cardiovascular en los pacientes en hemodiálisis, hace necesario profundizar en el estudio de la incidencia de arteriopatía periférica y amputaciones en estos pacientes.

Objetivos: identificar y analizar la incidencia de amputaciones no traumáticas, gangrenas, úlceras, y claudicación intermitente, determinando que factores aterogénicos son los que más inciden en estas lesiones.

Método: se estudió en 170 pacientes en programa de diálisis de la provincia de Cáceres, 46 diabéticos y 124 no diabéticos, los siguientes factores aterogénicos: edad, sexo, características de la diabetes, hiperlipidemia, y PTH.

Resultados: presentaban claudicación intermitente 33 pacientes, úlceras 14, gangrena 2, y amputaciones 11. La diabetes es el factor de mayor incidencia de la enfermedad, unido a la HTA, sexo, edad, y elevadas concentraciones de PTH. La hipertensión es más aterogénica cuando se mantiene tras el inicio de diálisis. La documentación de

Correspondencia:

José María Lancho Casares

Sección de Nefrología- Unidad de Hemodiálisis

Hospital San Pedro de Alcántara

Av Pablo Naranjo s/n

10003 Cáceres

lanchocasares@terra.es enfermería no recoge datos de la enfermedad ni de su evolución.

Es necesario planificar estrategias que prevengan la aparición de arteriopatía periférica y establecer registros de enfermería con relación a la enfermedad, inspección y exploración de los miembros inferiores, identificando los factores de riesgo para establecer los cuidados específicos e información y educación al paciente

PALABRAS CLAVE:

- HEMODIÁLISIS

- ARTERIOPATÍA PERIFÉRICA

- FACTORES DE RIESGO

\section{Patient on dialysis programme and peripher-} al arteriopathy

\section{Abstract}

The high prevalence of cardiovascular disease in haemodialysis patients makes it necessary to study in more depth the incidence of peripheral arteriopathy and amputations in these patients.

Objectives: to identify and analyse the incidence of non-trauma related amputations, gangrene, ulcers and intermittent claudication, determining which atherogenic factors have the greatest incidence on these injuries.

Method: the following atherogenic factors were studied in $\mathbf{1 7 0}$ patients on a dialysis programme in 
the province of Cáceres, 46 diabetic and 124 nondiabetic: age, sex, characteristics of the diabetes, hyperlipidaemia and PTH.

Results: 33 patients presented intermittent claudication, 14 presented ulcers, 2 gangrene and 11 amputations. Diabetes is the factor with the greatest incidence on the disease, together with hypertension, sex, age and high concentrations of PTH. Hypertension is more atherogenic when maintained after the commencement of dialysis. The nursing documentation does not reflect data on the disease or its evolution.

It is necessary to plan strategies that prevent the appearance of peripheral arteriopathy and to establish nursing records in relation to the disease, inspection and examination of lower limbs, identifying the risk factors in order to establish the specific care required and patient information and education

\section{KEY WORDS \\ - HAEMODIALYSIS \\ - PERIPERHAL ARTERIOPATHY \\ - RISK FACTORS}

\section{Introducción}

La prevalencia de la enfermedad cardiovascular en pacientes en diálisis es muy alta e incluye en muchos casos la aparición de arteriopatía periférica (AP) lo que puede conllevar una amputación. En la población general es conocido que la tasa de de amputación de miembros inferiores varía según la localización geográfica y raza ${ }^{1}$. La arteriopatía periférica afecta a 22 millones de personas en Norteamérica y Europa

En general, la incidencia de las AP es más frecuente en el hombre, apareciendo en la mujer diez años más tarde, y en ambos sexos aumenta con la edad. Entre los varones de 35 a 44 años la incidencia es de un $1 \%$, mientras que en $>65$ años es de un $6 \%$. Uno de cada diez varones mayores de 75 años padece AP, clínicamente significativa.
Los factores de riesgo relacionados con la AP y las amputaciones de miembros inferiores son los ya conocidos, tanto en la población general como en los pacientes en diálisis, considerándose como más significativos la diabetes, las alteraciones vasculares, la edad, la hipertensión y el tabaquismo².

La información epidemiológica sobre la arteriopatía periférica de los enfermos en diálisis puede ser insuficiente o contradictoria, aunque publicaciones recientes muestran la elevada incidencia de amputación de miembros inferiores por causas no traumáticas en estos pacientes ${ }^{1}$.

La prolongación de esperanza de vida y el aumento de la prevalencia de la insuficiencia renal crónica, secundaria a nefropatía diabética y etiología vascular hacen que asistamos desde hace años a la entrada en programa de tratamiento sustitutivo de pacientes sin límite de edad y con la patología asociada a riesgo vascular, fundamentalmente diabéticos. Esto se asocia a un aumento importante de la morbilidad de origen cardiovascula ${ }^{2,3}$ ya que el aumento de arteriopatía periférica incrementa el riesgo de muerte de 3 a 6 veces en las formas asintomáticas y hasta 15 veces en las formas severas.

En España, un estudio sobre 516 pacientes en hemodiálisis, reflejó una elevada prevalencia de amputaciones de miembros inferiores, por causas no traumáticas, siendo un 4,5\% menor que la observada por un estudio en Estados Unidos. Estudios en hospitales españoles indican que hasta un 36\% de estos pacientes presentaban patología vascular ${ }^{4}$.

En Extremadura, en el año 2004, la edad media de entrada en tratamiento sustitutivo fue de 64 años en varones (rango 89-17), siendo ligeramente superior en las mujeres (rango 85-29). En Cáceres la edad media del paciente en programa sustitutivo es de 67 años, siendo la más alta en el área de Navalmoral con 71 años, seguida de la de Plasencia con 69 años $^{5}$. Este único hecho ya nos hacer prever que los problemas vasculares estarán presentes en nuestras unidades.

\section{Objetivos}

1. Determinar y analizar la prevalencia e incidencia de la arteriopatía periférica, amputaciones no traumáticas, gangrenas, úlceras y claudicación intermitente en todos los pacientes de la provincia 
de Cáceres en tratamiento sustitutivo con hemodiálisis.

2. Determinar que factores aterogénicos son los que inciden más significativamente en el desarrollo de AP.

\section{Material y Método}

Analizamos a todos los pacientes incluidos en programa de hemodiálisis en la provincia de Cáceres: en el hospital San Pedro de Alcántara, y en los centros concertados Fresenius M.Care Extremadura de Cáceres y Plasencia. Los datos fueron recogidos por las enfermeras de los centros citados. Previamente se elaboró un documento para la recogida de datos en el que se incluyeron variables relacionadas con riesgo aterogénico, como edad, sexo, tiempo de permanencia en tratamiento sustitutivo, enfermedad base, HTA, antecedentes vasculares, hiperlipidemias, calcio, fósforo, PTH, presencia de calcificaciones, claudicación, úlceras, gangrena y amputaciones. También se recogieron otros datos que indicaban riesgo de padecer lesiones isquémicas, como la enfermedad de uñas, la deformidad de los dedos, callos o durezas.

Las cifras de TA se recogieron antes de la entrada al programa de diálisis y tras su inicio. Los parámetros analíticos fueron recogidos del último control. No se considero el consumo de tabaco ya que un sondeo previo demostró que sólo 4 pacientes eran fumadores.

Se realizó una valoración de los datos recogidos en los registros de enfermería de cada paciente: historia de enfermería, hoja de evolución, hoja de registros de diálisis y datos sobre información de la patología vascular del paciente.

Se incluyó una guía de orientación para cumplimentar los registros. El análisis estadístico se realizó a partir de una base de datos analizada por el programa SPSS 13.0. Relacionamos la presencia de la arteriopatía periférica con cada uno de los factores de riesgo vascular.

\section{RESULTADOS}

1.- Fueron incluidos todos los pacientes de la provincia de Cáceres en programa de hemodiálisis; un total de 170 pacientes. La población considerada tiene las características que se reflejan en la tabla 1. Presentaba claudicación intermitente 33 pacientes, úlceras 14, gangrena 2, y 11 amputaciones ( 5 de ambos miembros).

Hombres: 91 (53.5\%), mujeres: 79 (46,5\%).

Edad media: 65,94 años (rango 21- 90 años).

Tiempo medio en programa de HD: 3,78 años (rango 1 mes-21 años)

Etiología de la IRC:

- Diabetes Mellitus: tipo II (27,02\%).

- No filiadas: 36 (21.1\%)

- Glomerulonefritis: 34 (20\%)

- Vascular: $14(8.2 \%)$

- Pielonefritis: $10(5.8 \%)$

- Poliquistosis: 9 (5.2\%)

- Otras: 21 (12.3\%)

Tabla 1. Características de los pacientes

2.- De todos los pacientes $98(57,7 \%)$ tenían diferentes deformidades en dedos de los pies, callos, helomas y enfermedades de las uñas (onicomicosis, engrosamientos de las uñas de pie), y durezas. Dos pacientes tenían un gran engrosamiento de placa ungueal. Se ha de destacar que todos diabéticos que no estaban amputados tenían hongos en las uñas.

3.- Las relaciones entre las manifestaciones clínicas del daño vascular y los diferentes parámetros estudiados son las siguientes:

a) Edad: los pacientes de mayor edad desarrollaron más lesiones en todos los apartados (claudicación, úlceras, gangrenas, y amputaciones) (figura 1). 


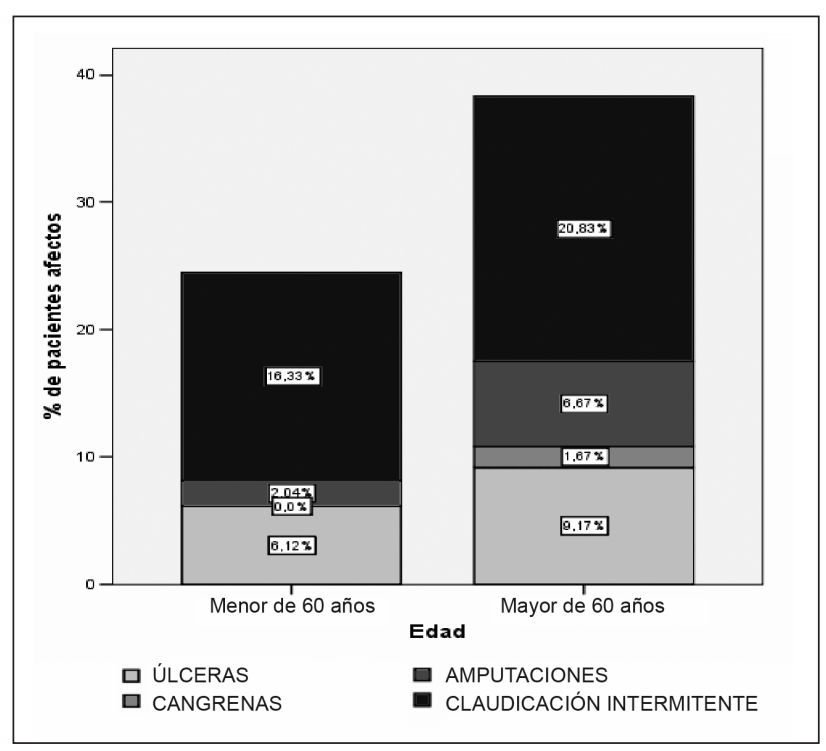

Figura 1. Relación entre edad y alteraciones vasculares

b) Sexo: los hombres presentaron más lesiones isquémicas en todos los apartados (figura 2)

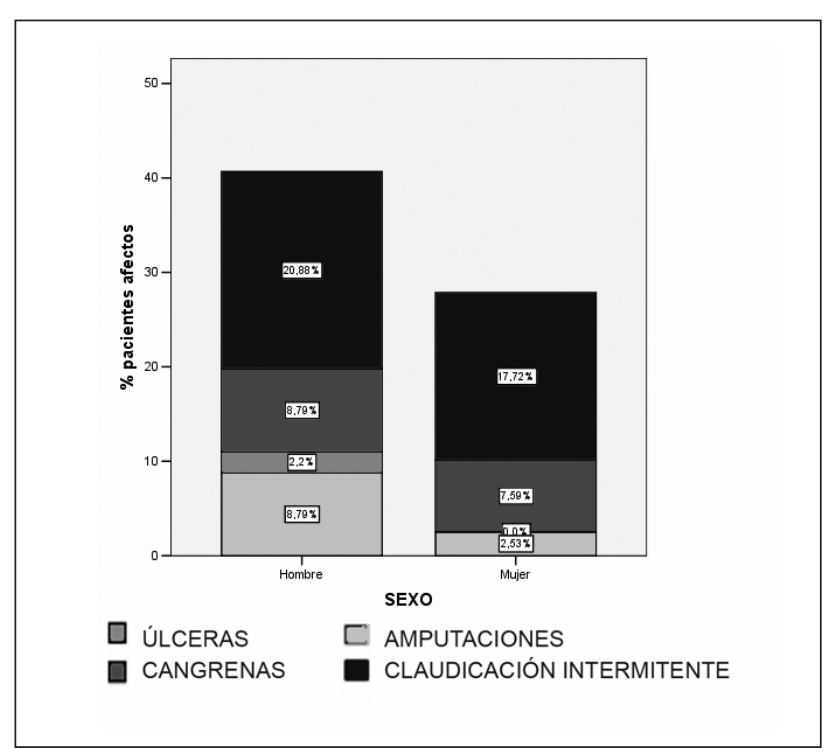

Figura 2. Relación entre sexo y alteraciones vasculares

\section{c) Pacientes diabéticos}

- De los 46 pacientes diabéticos (27.05\% del total de pacientes). Son 22 hombres y 24 mujeres. Sufrieron amputaciones 6 (5 hombres y 1 mujer). Fueron amputados de los dos miembros inferiores 5 y uno de un sólo miembro. Las amputaciones fueron su- pracondileas y de los dos miembros, a excepción de una mujer que fue del antepié.

- Presentaban gangrena 2

- Úlceras en los pies 10

- Claudicación intermitente 10

\section{d) Pacientes no diabéticos}

- No eran diabéticos 124 pacientes $(73 \%)$

- Amputaciones 4

- Úlceras 4

- Claudicación intermitente 23 (16,9\%)

e) Hipertensión (al inicio y después de la entrada en programa de hemodiálisis): se consideró hipertensos (180/85) a los pacientes que presentaban hipertensión previa a la entrada de diálisis, una vez iniciado el tratamiento sustitutivo o que estuviesen en tratamiento de hipotensores. Eran hipertensos $100(58,8 \%)$ antes de la entrada en programa sustitutivo y presentaba las siguientes alteraciones (figura 3):

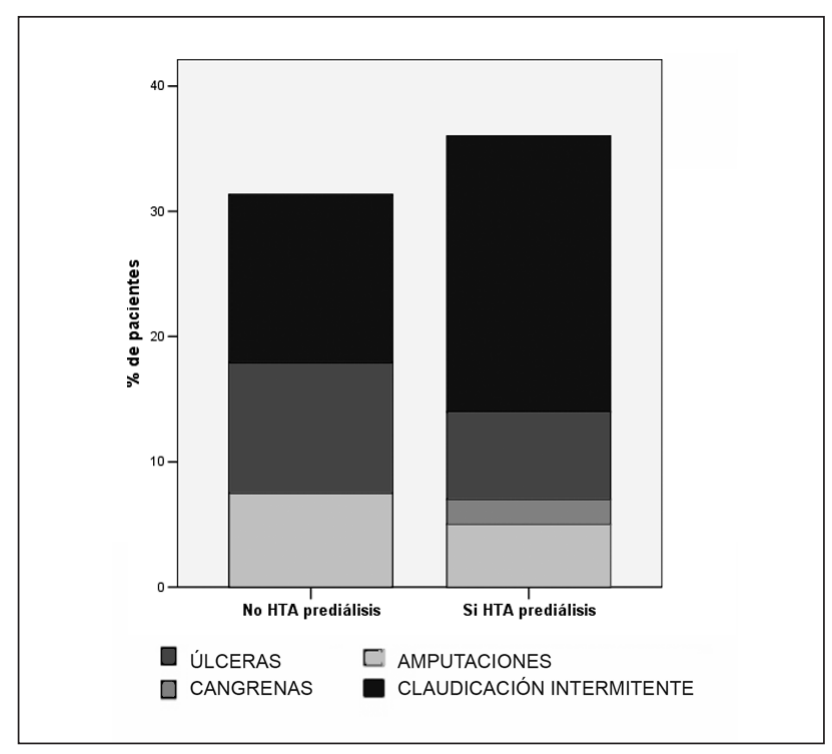

Figura 3. Relación entre HTA predialisis y alteraciones vasculares

- Amputaciones 8

- Úlceras 10

- Gangrena 3

- Claudicación intermitente 22

De estos pacientes 74 normalizaron sus cifras de tensión arterial tras su entrada en tratamiento sustitutivo. Los pacientes que estando ya en programa de 
hemodiálisis iniciaron hipertensión o se mantenían hipertensos desarrollaron lesiones isquémicas mucho más que los pacientes normotensos después del inicio en programa de hemodiálisis (figura 4)

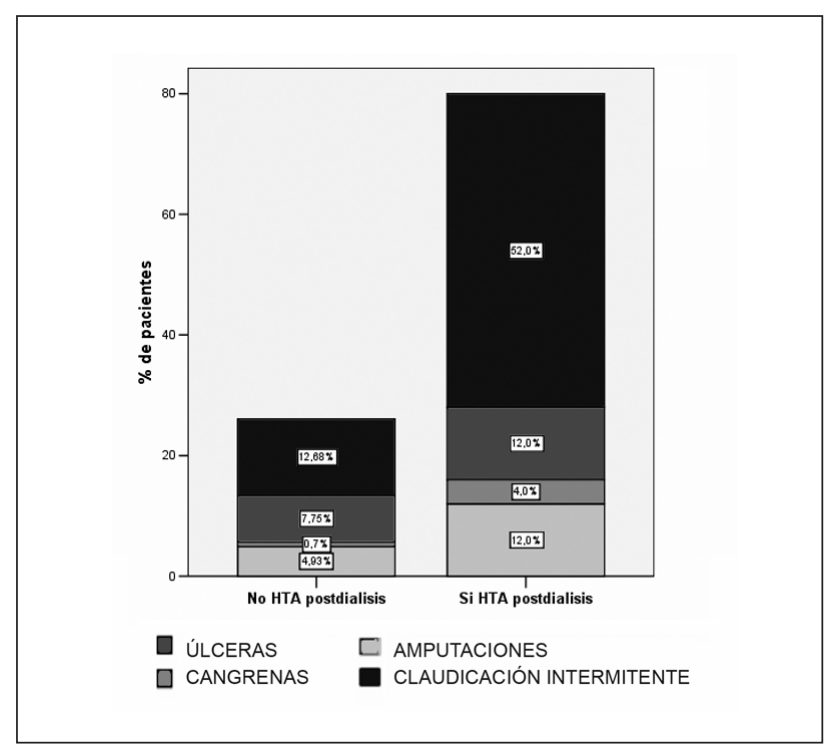

Figura 4. Relación entre HTA en tratamiento y alteraciones vasculares

f) Hiperlipidemia. Presentan cifras de colesterol > $180 \mathrm{mg} 21$ pacientes ( $12.4 \%$ ), en estos se observan las siguientes alteraciones:

- Amputaciones 2

- Úlceras 2

- Claudicación intermitente 1

Presentaban cifras de triglicéridos $>125 \mathrm{mg}, 63 \mathrm{pa}-$ cientes y en ellos se observan las siguientes alteraciones:

- Amputaciones 4

- Úlceras 7

- Claudicación intermitente 1

g) PTH. Considerando el valor de referencia < de 50, 140 pacientes (el $82 \%$ ) tenían la PTH elevada y de ellos 55 la tenían superior a 300, con una cifra máxima de 2800; se observan las siguientes alteraciones:

- Amputaciones 8

- Úlceras 11

- Claudicación intermitente 33

- Gangrena 2

4.- En cuanto a la documentación y registros de enfermería, sólo la historia de enfermería tiene la posibilidad de recogida de datos referentes a AP; a veces se comenta en la hoja de evolución, describiendo lesiones y cuidados y sólo se recogieron en el $12 \%$ de las 170 historias y hojas de evolución. No existen protocolos de cuidados de lesiones, ni programas informativos de educación sanitaria al paciente. Desde noviembre 2006 existe un díctico informativo de prevención y cuidados de los pies en HD.

\section{Discusión y Conclusiones}

El resultado de este estudio muestra una elevada incidencia de la enfermedad vascular periférica, y así mismo un alto número de amputaciones por causas no traumáticas, claudicación intermitente y úlceras. Como señalan otros autores la diabetes es uno de los factores de riesgo más prevalentes ${ }^{4,5}$ y la afectación relacionada con la AP en pacientes diabéticos se triplica porcentualmente en todos los parámetros estudiados con respecto a los pacientes no diabéticos, además de la influencia del sexo, la edad, los antecedentes vasculares, la HTA, y las altas cifras de triglicéridos y PTH. Las deformidades y alteraciones de la piel y uñas estuvo presente en casi todos los pacientes, por tanto el cuidado e higiene de los pies debe ser un requisito imprescindible para evitar lesiones ${ }^{6}$.

En cuanto a la presencia de HTA se ha de destacar que los hipertensos "resistentes" al tratamiento con fármacos y hemodiálisis presentaron muchas más lesiones isquémicas que los pacientes que normalizaron sus cifras tensionales. Un porcentaje alto de pacientes mostraron colesterol igual o por debajo del rango de referencia (mala nutrición) y tuvieron más lesiones isquémicas. Por otra parte, la afectación vascular periférica es menor en pacientes con cifras de colesterol por encima de los límites que en pacientes con hipercolesterolemia a causa de mal nutrición. En todos los amputados las cifras de colesterol eran elevadas. La elevación de triglicéridos y de PTH también influye en la aparición de lesiones.

Aunque el consumo de tabaco influye en la aparición de lesiones ${ }^{6}$ en nuestro estudio el tabaquismo no se ha tenido en cuenta ya que sólo cuatro de los pacientes estudiados eran fumadores. 
Aunque enfermería tiene una papel fundamental en la prevención y detección precoz de lesiones isquemias ${ }^{7}$ en la documentación de enfermería no se recogen suficientes registros sobre la $A P$, por lo que al planificar estrategias para prevenir la enfermedad y su evolución ${ }^{8}$ debe procurarse establecer en nuestra documentación registros de enfermería, con respecto a la arteriopatía periférica, que nos ayudará a detectar lesiones y protocolizar la inspección y exploración de los miembros inferiores, con el fin de identificar precozmente lesiones y factores de riesgos, y establecer los cuidados específicos. La educación e información al paciente, familiares y cuidadores se debe realizar de forma sistemática.

\section{Agradecimientos:}

A los compañeros de las tres unidades de hemodiálisis que recogieron datos y registros fundamentales para poder realizar el trabajo. Al Dr. L. Crespo, médico de nuestro hospital, que gracias a su colaboración pude realizar el estudio estadístico a través del programa SPSS 13.0. A ALCER-CÁCERES, en la persona de su presidente, D. José Antonio Sánchez Lancho, que ayudó en la criba de los datos y registro.

\section{Bibliografía}

1. Sánchez Perales MC, García MJ, Borrego Uriel FJ, Viedma G, Gil Pérez del Barrio JM, Borrego Liébana J, Pérez P. Incidencia y factores de riesgo de amputación no traumática de miembros inferiores en los pacientes en hemodiálisis. Nefrología 2005; 25 (4): 399-402.

2. Moreno Esteban B. La Diabetes Mellitus. Ed. CEA.1987.134-135.

3. Protocolo del estudio coparenal. SEN. WWW/senefro.org.

4. Montenegro J. Arterosclerosis y calcificaciones vasculares en la enfermedad renal crónica. Nephrology $2005 ; 2$.

5. Enfermos renales en tratamiento sustitutivo Extremadura. Publicación Año 2004 de Extremadura.

6. Menezo R et al. Factores de Riesgos vascular en pacientes en diálisis: su prevención implica conocimiento. En: Libro de comunicaciones del XXIII Congreso de la SEDEN; Sevilla 8-11 octubre. Barcelona: HOSPAL; 1998.

7. Moreno E. El tabaquismo en los pacientes de hemodiálisis. Prevalencia de consumo y actitudes. En: Libro de comunicaciones del XXIII Congreso de la SEDEN; Sevilla 8-11 octubre. Barcelona: HOSPAL; 1998.

8. Carrasco E, Esteban JM. Manejo de la isquemia arterial en miembros inferiores en atención primaria. Grupo. Ferrer. Edikamed; 2004. 